\title{
Can we predict the presence of coronary lesions from blood pressure measurement? A new clinical method
}

\author{
Mohammad El Tahlawi, Mohammad Abdelbaset, Mohammad Gouda and Ikhlas Hussein
}

The roles of arterial function and structure in cardiovascular physiology have expanded with the development of a variety of parameters that evaluate arterial stiffness. Markers of arterial stiffness have been correlated with cardiovascular outcomes. We aimed to find a simple, clinical, noninvasive method to predict atherosclerosis that leads to the development of coronary artery disease (CAD). We aimed to find a simple, clinical, noninvasive method to predict atherosclerosis that leads to the development of CAD. We included 100 cases that underwent coronary angiography in our center owing to different indications. The blood pressure in all cases was measured by two different observers. The oscillatory systolic blood pressure (OSBP) was defined as the point at which the mercury began to oscillate to a minimum level of $1 \mathrm{~mm} \mathrm{Hg}$. The auscultatory systolic blood pressure (AUSBP) was defined as the first Korotkoff sound. The difference between OSBP and AUSBP was calculated and called the oscillatory gap (OG). The correlation between the $O G$ and the presence of coronary lesion in coronary angiography was statistically calculated. The study populations had a mean age of $57.3 \pm 9$ years. The mean \pm s.d. OG was $14.44 \pm 10.44$. There was a highly significantly positive correlation between the OG and the presence of coronary artery lesions $(r=0.399$ and $P$-value $<0.000)$. There was also a significantly positive correlation between the presence of hypertension and the OG $(r=0.376$ and $P$-value $<0.000$ ). The difference between OSBP and AUSBP could be used as a simple method to detect atherosclerotic arterial changes. This method could indicate the degree of arterial stiffness. There was a significantly positive correlation between this new indicator of arterial stiffness and the presence of CAD. Any patient with a wide gap between OSBP and AUSBP should be treated early with antihypertensive drugs and statins before the development of CAD.

Hypertension Research (2015) 38, 260-263; doi:10.1038/hr.2014.170; published online 8 January 2015

Keywords: atherosclerosis; blood pressure; coronary; coronary lesion; oscillatory blood pressure measurement

\section{INTRODUCTION}

The roles of arterial function and structure in cardiovascular physiology have expanded with the development of a variety of parameters that evaluate arterial stiffness. Measurements of arterial stiffness have clarified the results of clinical trials, demonstrating differing impacts on clinical outcomes, despite similar reductions in blood pressure (BP). ${ }^{1}$

There are significant associations between aortic atherosclerosis and major cardiovascular events. In particular, thoracic aortic atherosclerosis is closely related to the degree of coronary artery disease (CAD). ${ }^{2}$

With advancing polyvascular atherosclerosis, the accumulation of atherosclerosis risk factors, previous atherothrombotic events and more severe CAD are observed. ${ }^{3}$

There are several terms that characterize vascular wall properties: distensibility, elasticity, compliance and stiffness. The large number of parameters that can be utilized in studying large artery dynamics are the result of the pulsatile character of blood flow and the complex structure of the arterial wall. ${ }^{4}$
One of these parameters, arterial stiffness, can be measured using devices such as the Colins system (Colin Corporation, Hayashi, Komaki-city, Japan) and the SphygmoCor system (AtCor Medical, West Ryde, NSW, Australia). ${ }^{5}$

We attempted to find a simple clinical parameter that could predict atherosclerosis and, therefore, CAD. We hypothesized that the arterial column started to oscillate before Kortokoff's sound was heard. This gap increased with the advancing of arterial atherosclerosis. Hence, this gap could predict cardiovascular atherosclerotic diseases, regardless of the presence of hypertension.

\section{METHODS}

Study design

This was a cross-sectional study enrolling 100 patients who underwent coronary angiography at Zagazig University Hospital owing to different indications. We included all age groups and both sexes.

\section{Ethical approval}

The study was approved by the Ethical Committee of the Faculty of Medicine, Zagazig University. Written informed consent was obtained from all of the patients. 


\section{BP measurement}

$\mathrm{BP}$ pressure was measured in all the cases. Brachial BP was measured by two trained different observers according to a highly standardized protocol, using a mercury sphygmomanometer with an appropriately sized cuff. One sphygmomanometer was placed on the right arm, and after at least $5 \mathrm{~min}$ of rest in the sitting position, three BP measurements were obtained, allowing for a $1 \mathrm{~min}$ interval between measurements. In cases of consistent systolic BP differences between arms, the arm with the higher BP value was used. The mercury was reduced by $2 \mathrm{~mm} \mathrm{Hg} \mathrm{s}^{-1}$. Oscillatory systolic blood pressure (OSBP) was defined as the point at which the mercury started to oscillate before the auscultation of any sounds. The first oscillation was considered if the mercury oscillated to a level of at least $1 \mathrm{~mm} \mathrm{Hg}$. This point was before the first Kortokoff sound (Figure 1). Auscultatory systolic blood pressure (AUSBP) was defined as the first Korotkoff sound. The difference between OSBP and AUSBP was calculated and called the 'oscillatory gap' (OG). All of the patients underwent coronary angiography owing to the presence of objective evidence of ischemia. The results of coronary angiography were recorded for all of the patients and were tabulated. The patients were classified according to the presence or absence of significant coronary lesions, defined as the presence of narrowing of the coronary vessel diameter $\geqslant 50 \%$ of the normal vessel diameter. The correlation between the difference between OSBP and AUSBP (OG) and the presence of coronary lesions in coronary angiography was statistically calculated.

\section{Statistical analysis}

Continuous variables are summarized as mean \pm s.d.s. Bivariate correlations were assessed between variables; then, the independent $t$-test was performed between variables based on the presence or absence of CAD. All of the calculations were performed using the SPSS software program, version 17.6

\section{RESULTS}

This study recruited 100 patients who underwent coronary angiography owing to different indications, with a mean age \pm s.d. of $57.3 \pm 9$ years. The cohort included 54 patients (54\%) with hypertension, $30(30 \%)$ with diabetes mellitus (DM) and $49(49 \%)$ with current smoking status. They had an average OG of $14.45 \pm 10.4$ $\mathrm{mm} \mathrm{Hg}$ (Table 1). Sixty-six patients had significant coronary lesions $(66 \%)$ and $34(34 \%)$ did not have significant coronary lesion in

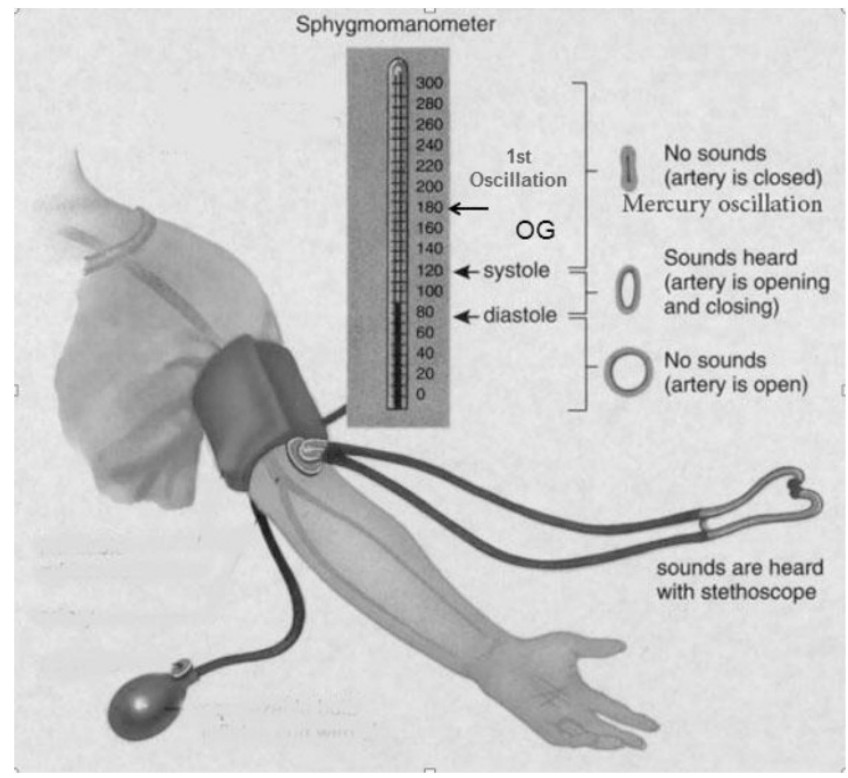

Figure 1 Illustration indicating the measurement of blood pressure with measurement of the oscillatory gap. A full color version of this figure is available at Hypertension Research online. coronary angiography. There was a highly significant difference between the two groups with and without significant coronary lesions regarding the presence of hypertension $(P=0.007)$, but there was no significant difference between both groups regarding the presence of diabetes or current smoking status (Table 2).

There was a highly significant difference between both groups with and without significant coronary lesions regarding the magnitude of OG $(P=0.000)$. There was a highly significant correlation between the magnitude of the $O G$ and the incidence of significant coronary lesion $(P<0.000, r=0.399)$ (Table 3$)$. There was also a significant correlation between the OG and the presence of systolic hypertension $(P<0.000, r=0.376)$ (Table 3). There was no significant relationship between the OG and the presence of DM or with the presence of a current history of smoking. There was a highly significant correlation between the OG and the age of the patients $(P=0.001, r=0.319)$ (Table 3).

\section{DISCUSSION}

In this study, we used a simple clinical method to predict the incidence of CAD. The difference between the oscillatory and auscultatory systolic pressures, called the 'oscillatory gap', could be a new clinical marker for the cascade of arterial atherosclerosis, which can be reflected in the coronary arteries. This possibility is strengthened by the presence of a highly positive correlation with hypertension.

This difference could be explained by the increased stiffness of the arterial system due to advanced atherosclerosis.

Increased elasticity (or stiffness, inverse of compliance) of the central elastic arteries is the primary cause of increased systolic and pulse pressure with advancing age and in patients with cardiovascular disease, including hypertension, and it is due to degeneration and hyperplasia of the arterial wall. As elasticity increases, the transmission velocity of both forward and backward (or reflected) traveling waves increases, causing the reflected wave to arrive earlier in the central aorta and augmenting pressure in late systole. ${ }^{7}$

Therefore, abnormal aortic function in hypertension has generally been attributed to the accelerated breakdown of elastin in the aorta, leading to dilatation of the lumen and stiffening of the wall as elastin is replaced with stiffer collagen. ${ }^{8}$

Thus, the stiffer the arterial system is, the greater the OG is. This finding could be explained by the fact that; when a material is not stressed in tension or compression beyond its elastic limit, its individual particles perform elastic oscillations. ${ }^{9}$

In addition, sound propagation is controlled by the rule that the speed of sound within a material is a function of the properties of the material and is independent of the amplitude of the sound wave. Hence, sound does travel at different speeds in different materials. ${ }^{10}$ Therefore, the propagation of the auscultatory first Kortokoff sound is more rapid in elastic arteries than in rigid and stiff ones. This is because there is positive relationship between the speed of sound in a solid and its density and elastic constants. ${ }^{10}$

Matter in a medium is periodically displaced by sound waves and thus oscillates. The energy carried by a sound wave converts back and forth between the potential energy of additional compression (in case of longitudinal waves) or lateral displacement strain (in case of transverse waves) of the matter and the kinetic energy of the oscillations of the medium.

The speed of sound depends on the medium through which the waves pass and is a fundamental property of the material. In general, the speed of sound is proportional to the square root of the ratio of the elastic modulus (stiffness) of the medium to its density. ${ }^{11}$ 
Table 1 Patient characteristics

\begin{tabular}{lcc}
\hline Parameter & Value & Percentage (\%) \\
\hline Age & $57.3 \pm 9$ years (range 34-79) & \\
Hypertension & 54 & $54 \%$ \\
Diabetes & 30 & $30 \%$ \\
Smoking & 49 & $49 \%$ \\
Oscillatory gap & $14.45 \pm 10.4 \mathrm{~mm} \mathrm{Hg}$ & \\
\hline
\end{tabular}

Table 2 Patient characteristics and OG in both groups of patients with and without coronary lesions

\begin{tabular}{|c|c|c|c|}
\hline Parameter & Number & Mean \pm s.d. & P-value \\
\hline \multicolumn{4}{|l|}{$O G$} \\
\hline With coronary lesion & 66 & $17.42 \pm 11.34 \mathrm{~mm} \mathrm{Hg}$ & 0.000 \\
\hline Without coronary lesion & 34 & $8.68 \pm 4.66 \mathrm{~mm} \mathrm{Hg}$ & \\
\hline \multicolumn{4}{|l|}{ Age } \\
\hline With coronary lesion & 66 & $58.29 \pm 9.07$ years & NS \\
\hline Without coronary lesion & 34 & $55.38 \pm 8.92$ years & \\
\hline \multicolumn{4}{|l|}{ Hypertension } \\
\hline With coronary lesion & 66 & & \\
\hline \multicolumn{4}{|c|}{34 Without coronary lesion0.007 } \\
\hline \multicolumn{4}{|c|}{ Diabetes } \\
\hline With coronary lesion & 66 & & NS \\
\hline Without coronary lesion & 34 & & \\
\hline \multicolumn{4}{|l|}{ Smoking } \\
\hline With coronary lesion & 66 & & NS \\
\hline Without coronary lesion & 34 & & \\
\hline
\end{tabular}

Abbreviations: NS, non significant; OG, Oscillatory gap.

The velocity of transmission and the propagation of vibrations in material media depend on the elasticity of such media and its inertia. ${ }^{12}$ Stiff structures tend to transmit vibrations at higher frequencies than compliant (un-stiff) structures. Stiff machines, with low internal or external damping, will transmit these vibrations throughout the structure. ${ }^{13}$ Hence, the vibration of blood in more rigid vessels causes the walls of these vessels to oscillate more than elastic vessels, which dampen these oscillations. These oscillations are reflected in the mercury earlier than the sound that is auscultated. This difference creates the OG.

Therefore, the rigidity of the artery is an important factor in creating this OG, regardless of its size because the cross-sectional area of the artery was not associated with increased arterial stiffness. ${ }^{10}$

This study found a highly significant correlation between the OG and the presence of systolic hypertension. This finding was consistent with several studies that demonstrated stiffness of the central arteries in hypertensive subjects. ${ }^{14,15}$ However, we believe that the OG might be a better marker than hypertension itself in predicting atherosclerosis, because this study showed that the OG could predict coronary lesions even in normotensive patients. This finding indicated that the OG might constitute an early stage before the development of hypertension.

Although the populations in this study had several risk factors for atherosclerosis, including hypertension (54\%), DM (30\%) and a
Table 3 Correlations between OG and age, CAD and HTN

$O G$

\begin{tabular}{ll}
\hline CAD & $r=0.399$ \\
& $P<0.000$ \\
HTN & $r=0.376$ \\
& $P<0.000$ \\
Age & $r=0.319$ \\
& $P<0.001$
\end{tabular}

Abbreviations: CAD, Coronary artery disease; HTN, Hypertension; OG, Oscillatory gap.

current history of smoking (49\%), there was a highly significant correlation only between OG and hypertension.

Several hypotheses have been proposed to explain the increased central aortic stiffness of patients with hypertension. In animal models of hypertension, increased deposition of collagen and elastin has been observed in the aortic wall, ${ }^{16}$ suggesting that changes in wall stiffness might provide the basis for abnormal vessel properties.

Increased aortic tone or myocyte hypertrophy in the setting of hyperactivity of the sympathetic nervous system or in response to elevated myogenic tone might be contributing factors. ${ }^{17}$

Alternatively, abnormal endothelial function might be involved in the functional imbalance between aortic flow and diameter. It is well accepted that the endothelium is capable of modulating smooth muscle mass and tone and therefore the diameters of the large and small blood vessels; this modulation could be lost because of atherosclerosis. $^{17}$

We found a significant correlation between this OG and age. Some studies have found that with normal aging, the stiffness of the central arteries increases. ${ }^{18}$ Therefore, the OG could be considered as a sign of arteriosclerosis that occurs with aging. However, the stiffness of peripheral arteries remains unchanged or decreases with age. ${ }^{19}$

This divergent change in peripheral vessel properties might moderate the abnormalities in total arterial compliance and pulsewave transmission that would otherwise result from deterioration in aortic properties. ${ }^{17}$

We can understand the highly significant correlation between the OG and the presence of CAD, because the structural and functional properties of the large arteries are important determinants of cardiovascular hemodynamics. ${ }^{20,21}$ These properties have important roles in the transformation of the pulsatile left ventricular stroke volume into continuous blood flow at the level of most of the peripheral vessels. This elastic system also allows for the maintenance of sufficient diastolic BP, which is essential for adequate coronary perfusion. ${ }^{4}$

Arterial stiffness has been identified as an independent risk factor for the development of $\mathrm{CAD}$, both in the general population and in people with established $\mathrm{CAD} .^{22}$

It has already been recognized that arterial stiffness, the main mechanism underlying pulse pressure widening, is an independent risk factor for cardiovascular disease. ${ }^{23}$

\section{CONCLUSION}

The study demonstrated a new and simple clinical test, 'the oscillatory gap', that indicates atherosclerosis, which can be used to predict the presence of CAD. Patients with wide OG should be treated early with drugs that improve arterial stiffness, such as antihypertensive drugs and statins. 
1 Liao J, Farmer J. Arterial stiffness as a risk factor for coronary artery disease. Curr Atheroscler Rep 2014; 16: 387.

2 Tresoldi S, Di Leo G, Zoffoli E, Munari A, Primolevo A, Cornalba G, Sardanelli F. Association of aortic wall thickness on contrast-enhanced chest CT with major cerebrocardiac events. Acta Radiol 2013; 55: 1040-1049.

3 Przewłocki T, Kabłak-Ziembicka A, Kozanecki A, Rzeźnik D, Pieniazek P, Musiałek P, Piskorz A, Sokołowski A, Rosławiecka A, Tracz W. Polyvascular extracoronary atherosclerotic disease in patients with coronary artery disease. Kardiol Pol 2009; 67: 978-984.

4 Baguet JP, Kingwell BA, Dart AL, Shaw J, Ferrier KE, Jennings GL. Analysis of the regional pulse wave velocity by Doppler: methodology and reproducibility. J Hum Hypertens 2003; 17: 407-412.

5 Youn JC, Kim JY, Park S, Kwon J, Lee HS, Shin DH, Lee SH, Kang SM, Hoon Son N, Jang $\mathrm{Y}$. Comparison of arterial stiffness indices measured by the Colins and SphygmoCor systems. Hypertens Res 2012; 35: 1180-1184.

6 Dean JA, Coulabier D. A word processing database and statistic program for epidemiology on microcomputer CDC, Atlanta, GA, USA, 2000.

7 Nichols WW, Edwards DG. Arterial elastance and wave reflection augmentation of systolic blood pressure: deleterious effects and implications for therapy. J Cardiovasc Pharmacol Ther 2001; 6: 5-21.

8 O'Rourke M. Mechanical principles in arterial disease. Hypertension 1995; 26: 2-9.

9 NDT Education Resource Center, 2001-2012, The Collaboration for NDT Education, lowa State University https://www.nde-ed.org/EducationResources/CommunityCollege/ Ultrasonics/Physics/elasticsolids.htm. Accessed 29 March 2014.

10 Otsuka T, Munakata R, Kato K, Kodani E, Ibuki C, Kusama Y, Seino Y, Kawada T. Oscillometric measurement of brachial artery cross-sectional area and its relationship with cardiovascular risk factors and arterial stiffness in a middle-aged male population. Hypertens Res 2013; 36: 910-915.

11 Wikipedia. Sound. http://en.wikipedia.org/wiki/Sound. Accessed 28 August 2014.

12 Oscillation Waves and Acoustics, chapter 7. International publishing house Pvt Ltd, 2010, P261I.K.
13 Stratulat Florin. Comparative study of a mill machine's dynamics. UPB Sci Bull, Series C 2009; 71: 45-60.

14 Avolio AP, Deng FQ, Li WQ, Luo YF, Huang ZD, Xing LF, O'Rourke MF. Effects of aging on arterial distensibility in populations with high and low prevalence of hypertension: comparison between urban and rural communities in China. Circulation 1985; 71: 202-210.

15 Boutouyrie P, Laurent S, Benetos A, Girerd XJ, Hoeks AP, Safar ME. Opposing effects of ageing on distal and proximal large arteries in hypertensives. J Hypertens Suppl 1992; 10: S87-S91.

16 Wolinsky H. Long-term effects of hypertension on the rat aortic wall and their relation to concurrent aging changes: morphological and chemical studies. Circ Res 1972; 30: 301-309.

17 Mitchell GF, Lacourcière Y, Ouellet JP, Izzo JL Jr, Neutel J, Kerwin LJ, Block AJ, Pfeffer MA. Determinants of elevated pulse pressure in middle-aged and older subjects with uncomplicated systolic hypertension: the role of proximal aortic diameter and the aortic pressure-flow relationship. Circulation 2003; 108: 1592-1598.

18 Learoyd BM, Taylor MG. Alterations with age in the viscoelastic properties of human arterial walls. Circ Res 1966; 18: 278-292.

19 van der Heijden-Spek JJ, Staessen JA, Fagard RH, Hoeks AP, Boudier HA, van Bortel LM. Effect of age on brachial artery wall properties differs from the aorta and is gender dependent: a population study. Hypertension 2000; 35: 637-642.

20 Isnard RN, Pannier BM, Laurent S, London GM, Diebold B, Safar ME. Pulsatile diameter and elastic modulus of the aortic arch in essential hypertension: a noninvasive study. J Am Coll Cardiol 1989; 13: 399-405.

21 Nichols WW, O'Rourke MF. McDonald's Blood Flow in Arteries: Theoretical, Experimental and Clinical Principles, 4th edn. Arnold: London, UK, 1998.

22 Veerasamy M, Ford GA, Neely D, Bagnall A, Macgowan G, Das R, Kunadian V. Association of aging, arterial stiffness and cardiovascular disease: a review. Cardiol Rev 2014: 22: 223-232.

23 Franklin SS, Larson MG, Khan SA, Wong ND, Leip EP, Kannel WB, Levy D. Does the relation of blood pressure to coronary heart disease risk change with aging? The Framingham Heart Study. Circulation 2001; 103: 1245-1249. 